\title{
A REAL TIME MUSIC SYNTHESIS ENVIRONMENT DRIVEN WITH BIOLOGICAL SIGNALS
}

\author{
Burak Arslan ${ }^{1}$, Andrew Brouse ${ }^{2}$, Julien Castet $^{3}$, Remy Léhembre $^{4}$, Cédric Simon $^{4}$, Jean Julien Filatriau ${ }^{4}$, Quentin Noirhomme $^{4 *}$ \\ ${ }^{1}$ TCTS Lab Faculté Polytechnique de Mons, Mons, Belgium \\ ${ }^{2}$ Computer Music Research, University of Plymouth, Drake Circus, Plymouth, U.K., \\ ${ }^{3}$ Polytechnics National Institute of Grenoble, Grenoble, France, \\ ${ }^{4}$ TELE Lab, Université catholique de Louvain, Louvaine-la-Neuve, Belgium.
}

\begin{abstract}
This study proposes to use the analysis of physiological signals (electroencephalogram (EEG), electromyogram (EMG), heart beats etc) to control sound synthesis algorithms in order to build biologically driven musical instruments. A real time music synthesis environment and algorithms are developed to map these signals into sound. Finally, a "bio-orchestra", with two new digital musical instruments controlled by the EEGs and EMGs of two bio-musicians demonstrated this concept with a live concert on stage.
\end{abstract}

\section{INTRODUCTION}

Advances in computer science and specifically in Human-Computer Interaction (HCI) have enabled musicians to use sensor-based computer instruments to perform music [1]. Musicians can now use data from different sensors (that reflect cardiac, or muscle activity or limb position etc.) to control sound [2]. Simultaneously, Brain-Computer Interface (BCI) research has shown that cerebral patterns can be used as a source of control [3]. Indeed, cerebral and conventional sensors can be used together [4] with the aim of producing a 'body-music' controlled according to the musician's imagination and proprioception.

Some research has already been done toward integrating BCI and sound synthesis with two very different approaches. The first approach is the sonification of the data [5]. This process can be viewed as a translation of physiological signals into sound. The second approach aims to build a musical instrument [6]. In this case, the musician tries to use his physiological signals to control intentionally the sound production. We did choose the second approach essentially and decided to use the sonification for additional signals to enrich the acoustical content.

In the following, we present the architecture we developed to acquire, process and play music based on biological signals. Next the signal acquisition and signal processing techniques are explained and the results are given. We conclude with a brief discussion of the sound synthesis implementation along with the instruments we built.

\section{ARCHITECTURE}

We intend to build a robust architectural framework that could be reused with other biological data, other methods of analysis and other types of instruments. Therefore the signal acquisition, the signal processing and the sound synthesis are operated on different virtual machines that communicate by the network (Fig. 1). The data from different modalities are recorded on different machines.

\footnotetext{
*Quentin Noirhomme is supported by a grant from the Région Wallonne.
}

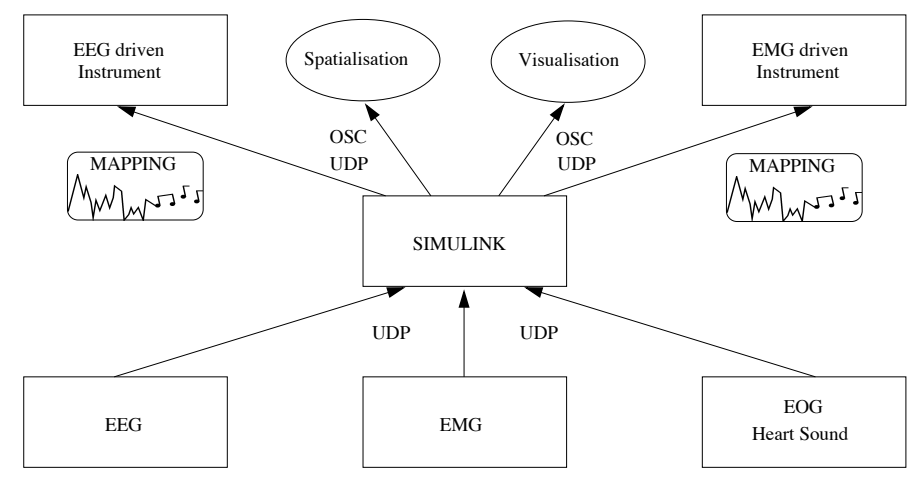

Fig. 1. System architecture

Once acquired, the data are sent to a Simulink program. Then they are processed before being sent to the musical instruments, and the sound spatialization and visualization routines via Open Sound Control. The musical instruments are build with Max/MSP. Below is an outline of the main software and data exchange architecture.

\subsection{Software}

Biosignal analysis is achieved with various methods including wavelet analysis and spatial filtering. Due to the flexibility of Matlab programming, all the algorithms are written in Matlab code whereas the signal acquisition from the EEG cap is made in C++. In order to better adapt to variabilities between subjects, a Simulink block diagram is developed that uses Level-2 M file S-functions with tuneable parameters. For visualization purposes, the virtual reality toolbox is used that allowed a feedback helping the user to control his/her EEG.

Max/MSP is a widely accepted and supported software programming environment optimized for flexible real-time control of music systems. We used it to build the musical instruments that use the recorded and processed biological signals

\subsection{Data Exchange}

Data are transferred from one machine to another with the UDP protocol. We chose it mainly for its better real-time capability. To communicate with the musical instrument we use a specific protocol one level higher than UDP: open sound control (OSC). OSC was conceived as a protocol for the real-time control of computer music synthesizers over modern heterogeneous networks. Its development was informed by shortcomings experienced with the established MIDI standard and the difficulties in developing a more flexible protocol 


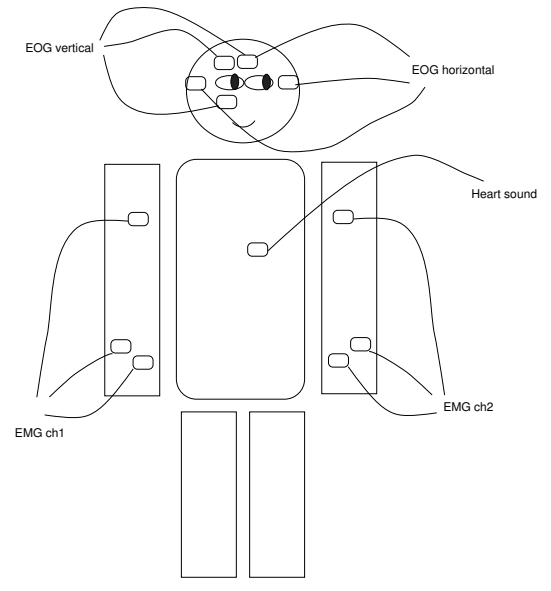

Fig. 2. Application of multiple electrodes and transducers

for effective real-time control of expressive music synthesis. Although it can function in principle over any appropriate transport layer such as WiFi, serial, USB or other data network, current implementations of OSC are optimized for UDP/IP transport over Fast Ethernet in a Local Area Network. For our project, we used OSC to transfer data from Matlab (running on a PC with either Linux or Windows OS) towards Max/MSP (running on a Macintosh OSX).

\section{DATA ACQUISITION}

Four types of signals are recorded with associated captors: EEG, EMG, heart sounds and EOG (see Fig. 1). EMG, EOG and heart sounds are acquired on one machine and EEG on another.

EEG data are recorded at $64 \mathrm{~Hz}$ on 19 channels with a DTI cap. Data are filtered between 0.5 and $30 \mathrm{~Hz}$. Channels are positioned following the 10-20 international system and $\mathrm{Cz}$ is used as reference. The subject sit in a comfortable chair and is asked to concentrate on different tasks. The recording is done in a normal office environment, e.g. a noisy room with people working, speaking and with music. The environment is not free from electrical noise as there are many computers, speakers, screen, microphones and lights around.

Disposable ECG electrodes were used for both EOG and EMG recordings. The sounds were captured using the Biopac BSL contact microphone. To record the EMG and heart sounds, three amplifiers of Biopac MP100 system were used. The amplification factor for the EMG was 5000 and the signals were filtered between 0.05-35 Hz. The microphone channel had 200 gain and DC-300 Hz bandwidth. Another 2 channel amplifier, ModularEEG is used to collect the EOG signals. This amplifier has 4000 gain and $0.4-60 \mathrm{~Hz}$ passband.

For real time capabilities, these amplified signals are fed to the National Instruments DAQPad 6052e analog-digital converter card that uses the IEEE 1394 port. Thus, the data can be acquired, processed and transferred to the musical instruments using Matlab environment and the Data Acquisition toolbox.

\section{BIOSIGNAL PROCESSING}

The aim of this work is to control sound and synthesize music using parameters derived from measured biological signals. We have tested different techniques to extract parameters giving meaningful control data to drive musical instruments. We mainly concentrated on the EEG (as it is the richest and most complex bio-signal) and EMG (easy control) signals.

For EMG, the data acquisition program samples blocks of data of $100 \mathrm{~ms}$ duration, and then analyzes this data. It calculates the energy and sends this information to the related instrument.

EEG analysis focuses on the detection of a users intent. It is based on the methods actually used in the BCI research (Fig. 3).

\subsection{Detection of User's Intent}

To detect different brain states we used the spatialisation of the activity and the different rhythms present in this activity. Indeed, each part of the brain has a different function and every subject may present specific rhythms at different frequencies. Three main rhythms are of great interest:

1. Alpha rhythm: usually between $8-12 \mathrm{~Hz}$, this rhythm describes the state of awareness.

2. Mu rhythm: This rhythm is also reported to range from 8 to $12 \mathrm{~Hz}$. The mu rhythm corresponds to motor tasks like moving the hands or legs, arms, etc. We use this rhythm to distinguish left hand movements from right hand movements.

3. Beta rhythm: Comprised of energy between $18-26 \mathrm{~Hz}$, the characteristics of this rhythm are yet to be fully understood but it is believed that it is also linked to motor tasks and higher cognitive function.

The well-known wavelet transform [7] is a suitable time-frequency analysis technique for the task detection. Each task can be detected by looking at specific band on specific electrodes. This operation, implemented with sub-band filters, provides us with a filter bank tuned to the frequency ranges of interest. We tested our algorithm on two subjects with different kinds of wavelets: Meyer wavelet, 9-7 filters, bi-orthogonal spline wavelet, symlet 8 and Daubechy 6 wavelets. We finally chose the symlet 8 which gave better overall results.

At the beginning we focused on eye blink detection and $\alpha$ band power detection since both are easily controllable by the musician. We then decided to use right and left hand movements by using two different techniques: Asymmetry ratio and spatial decomposition.

\subsubsection{Eye blinking and $\alpha$ band}

Eye blinking is detected on $\mathrm{Fp} 1$ and $\mathrm{Fp} 2$ electrodes in the $1-8 \mathrm{~Hz}$ frequency range by looking at increase of the band power. We process the signals from electrodes $\mathrm{O} 1$ and $\mathrm{O} 2$-occipital electrodes- to extract the power of the alpha band.

\subsubsection{Asymmetry ratio}

It is known that motor tasks activate the motor cortex area. Since the brain is divided into two hemispheres that control the two sides of the body it is possible to recognize when a person moves on the left or right side. Let $\mathrm{C} 3$ and $\mathrm{C} 4$ be the two electrodes positioned on the cortex, the asymmetry ratio can be written as:

$$
\Gamma_{F B}=\frac{P_{C 3, F B}-P_{C 4, F B}}{P_{C 3, F B}+P_{C 4, F B}}
$$

where $P_{C x, F B}$ is the power in a specified frequency band (FB), i.e. the mu frequency band. This ratio has values between 1 and -1 . Thus it is positive when the power in the left hemisphere (right hand movements) is higher than the one in the right hemisphere (left hand movements) and vice-versa. 


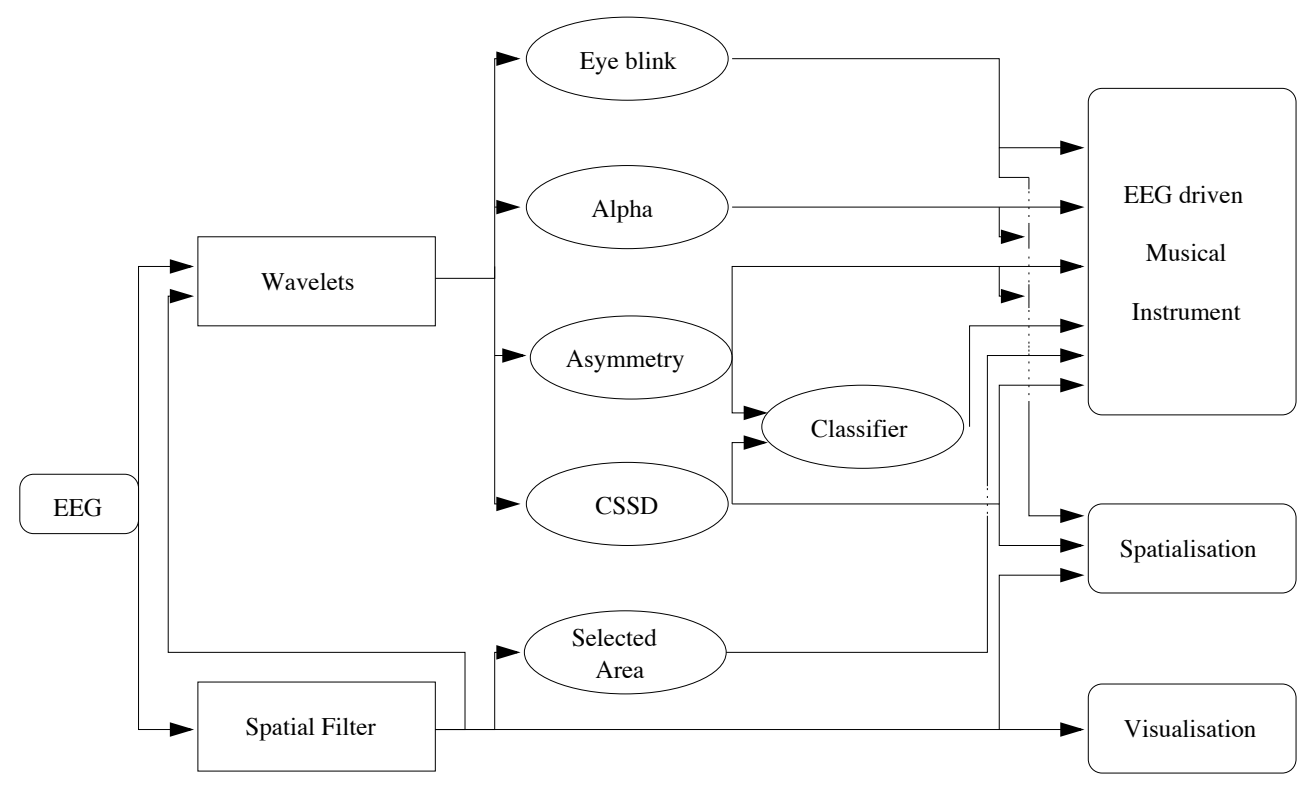

Fig. 3. EEG processing, from recording (left) to play (right).

\subsubsection{Spatial decomposition}

The asymmetry ratio cannot be used to distinguish more than two tasks. We used the Common Spatial Subspace Decomposition (CCSD) [8] method in that case. This method is based on the decomposition of the covariance matrix grouping two or more different tasks. Only the simple case of two tasks is explained below. It should be noted that this method needs a learning phase where the user executes the two tasks.

Let $X$ be a signal of dimension $N \times T$ for $N$ electrodes and $T$ samples. Decomposing $X$ in $X_{A}$ et $X_{B}, A$ and $B$ being two different tasks, we can obtain the autocovariance matrix for each task:

$$
R_{A}=X_{A} X_{A}^{T} \quad \text { and } \quad R_{B}=X_{B} X_{B}^{T}
$$

By extracting the eigenvectors and eigenvalues from $R$

$$
R=R_{A}+R_{B}=U_{0} \lambda U_{0}^{T}
$$

we can calculate the spatial factors matrix $W$ and the whitening matrix $P$ :

$$
W=U_{0} \lambda^{1 / 2} \quad \text { and } \quad P=\lambda^{-1 / 2} U_{0}^{T}
$$

If $S_{A}=P R_{A} P^{T}$ and $S_{B}=P R_{B} P^{T}$ then,

$$
S_{A}=U_{A} \Sigma_{A} U_{A}^{T} \quad S_{B}=U_{B} \Sigma_{B} U_{B}^{T}
$$

Matrices $U_{A}$ et $U_{B}$ are equal and the sum of their eigenvalues is equal to $1, \Sigma_{A}+\Sigma_{B}=I . \Sigma_{A}$ et $\Sigma_{B}$ can be written thus:

$$
\begin{aligned}
& \Sigma_{A}=\operatorname{diag}[\underbrace{1 \ldots 1}_{m a} \underbrace{\sigma_{1} \ldots \sigma_{m_{c}}}_{m c} \underbrace{0 \ldots 0}_{m b}] \\
& \Sigma_{B}=\operatorname{diag}[\underbrace{0 \ldots 0}_{m a} \underbrace{\delta_{1} \ldots \delta_{m_{c}}}_{m c} \underbrace{1 \ldots 1}_{m b}]
\end{aligned}
$$

Taking the first $m_{a}$ eigenvector from $U$, we obtain $U_{a}$ to compute the spatial filters $F$ and the spatial factors $G$ :

$$
F_{a}=W U_{a}
$$

$$
G_{a}=U_{a}^{T} P
$$

We proceed identically for the second task, taking this time the last $m_{b}$ eigenvectors. Specific signal components of each task can then be extracted easily by multiplying the signal with the corresponding spatial filters and factors. For the task A it gives:

$$
\hat{X}_{a}=F_{a} G_{a} X
$$

A support vector machine (SVM) with a radial basis function was used as a classifier.

\subsubsection{Results}

The detection of eye blinking during off-line and realtime analysis was higher than $95 \%$, with a $0.5 \mathrm{~s}$ time window. For hand movement classification with spatial decomposition, we chose to use a 2 s time window. A smaller window significantly decreases the classification accuracy. The CSSD algorithm needs more training data to achieve a good classification rate so we decided to use 200 samples of both right hand and left hand movements, each sample being a $2 \mathrm{~s}$ time window. Thus, we used an off-line session to train the algorithm.

We achieved an average of $90 \%$ good classifications during offline analysis, and $75 \%$ good classifications during real-time recording. Real-time recording accuracy was a bit less than expected. (This was probably due to a less-than-ideal environment - with electrical and other noise - which is not conducive to accurate EEG signal capture and analysis.) The asymmetry ratio gave somewhat poorer results.

\subsection{Sound Synthesis}

Artificial synthesis of sound is the creation, using electronic and / or computational means, of complex waveforms, which, when passed through a sound reproduction system can either mimic a real musical instrument or represent the virtual projection of an imagined musical instrument. Mapping is a significant problem in the case of biologically controlled instruments in that it is not possible to have 


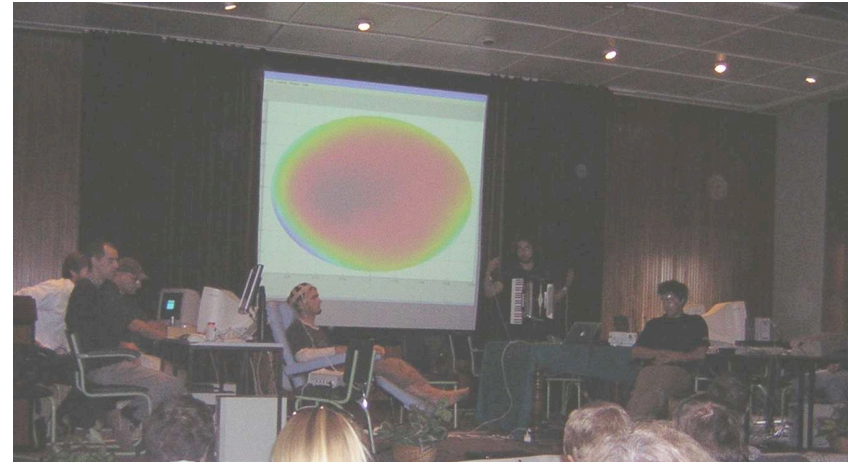

Fig. 4. Visualization of cortical activity during the live concert

an unambiguous interpretation of the meanings of biological signals whether direct or derived.

The first instrument developed in this study was a midi instrument based on additive synthesis and controlled by musician's electroencephalograms. There were two types of note trigger: First, the cycle of notes begins when the artist opens his eyes for the first time. The second control uses the EEG analysis: when the artist thinks about right or left body movements, he controls the direction of cycle rotation and the panning of the result. The succession of notes is subjected of two randomized variations, the note durations and the delta time between each note. The second instrument, driven by electromyograms of a second bio-musician, processed accordion samples recorded in live situation by granulation and filtering effects. Furthermore biological signals managed the spatialized diffusion over eight loudspeakers of sound produced by both previous instruments and the visual feedback. This was controlled by EEGs of the first bio-musician.

\subsection{Inverse problem and visualization}

In a musical performance, first comes the music. Nevertheless, visual data has an impact on it: How the musicians play their instruments, how they move and how they express their emotions. For the EMG driven instrument, the visual impact is achieved with the gestures of the musician, since these also are related to the sound generated (muscle contractions). In case of the EEG driven musical instrument, the musician must sit and stay immobile. To add a visual effect on the performance, we decided to use a projection of the EEG inverse problem solution. 361 current-dipoles were located on and oriented perpendicular to the cortex. Then the current in the dipoles was computed with the LORETA method [9]. Finally the current on every point of an half-sphere was interpolated from the solution and projected on a screen in the backstage(Fig.4).

\section{CONCLUSION}

This paper describes two musical instruments developed, that are driven by the biological signals of the musicians. One is based on the EEG and the other on the EMG. Although heart sounds and EOGs are recorded, they are not inserted to the instruments yet. This is left to further studies. One of the main achievements is the architecture we built. It enables the communication between any recording machine that can be linked to a network and a musical instrument. Since it is based on Matlab, any specific signal processing method can be easily implemented in the architecture. Furthermore the bridge built between Matlab and Max/MSP via Open Sound Control could be easily reused by other projects. Main signal processing is done on the EEG data although more elaborate techniques can be applied to various other biosignals in order to enrich the acoustical content. Using the algorithms on the realtime EEG data, high accuracy in controlling the synthesized musical sequence is obtained. This can be further improved by training the musicians to get familiar with the signals they generate. Finally, the whole concept is demonstrated to an audience in a live concert [10].

\section{REFERENCES}

[1] A. Tanaka, "Musical perfomance practice on sensor-based instruments," in Trends in Gestural Control of Music, M. M. Wanderley and M. Battier, Eds., pp. 389-406. IRCAM, 2000.

[2] Y. Nagashima, "Bio-sensing systems and bio-feedback systems for interactive media arts," in 2003 Conference on New Interfaces for Musical Expression (NIME03), Montreal, Canada, 2003, pp. 48-53.

[3] J. R. Wolpaw, N. Birbaumer, D. J. McFarland, G. Pfurtscheller, and T. M. Vaughan, "Brain-computer interfaces for communication and control," Clinical Neurophysiology, vol. 113, pp. 767-791, 2002, invited review.

[4] H. S. Lusted and R. B. Knapp, "Controlling computers with neural signals," Scientific American, pp. 82-87, November 1996.

[5] J. Dribus, "The other ear : a musical sonification of eeg data," in Proceedings of the 2004 International Conference on Auditory Display, Sydney, Australia, 2004.

[6] E. Miranda and A. Brouse, "Toward direct brain-computer musical interface," in 2005 Conference on New Interfaces for Musical Expression (NIME05), Montreal, Canada, 2005.

[7] S. Mallat, A wavelet tour of signal processing, Academic Press, 1998.

[8] P. Berg Y. Wang and M. Scherg, "Common spatial subspace decomposition applied to analysis of brain responses under multiple task conditions: a simulation study," Clinical Neurophysiology, pp. 604-614, November 1999.

[9] Roberto Domingo Pascual-Marqui, "Review of methods for solving the EEG inverse problem," International Journal of Bioelectromagnetism, vol. 1, no. 1, pp. 75-86, 1999.

[10] B. Arslan, A. Brouse, J. Castet, J.J. Filatriau, R. Lehembre, Q. Noirhomme, and C. Simon, "Biologically-driven musical instrument," in Proceedings of eNTERFACE'05 Summer workshop on multimodal interfaces, Mons, Belgium, 2005. 